Eur Surg Res 1995;27:427-428

\title{
Subject Index, Vol. 27, 1995
}

Adenylate energy charge 241 Adriamycin (doxorubicin) 301 Aging 250 Albendazole 340 Allopurinol 39,69,117,285 Animal model 277 Antioxidant(s) 69,292 Arterial ketone body ratio 77, 93 Ascorbate 39 Ascorbic acid 158

Bile duct ligation 332

- obstruction 127 Biliary drainage 11

- obstruction 137 Blood transfusion 19 Bone hemodynamics 197 Bowel resection 396 Breast cancer 346 Bronchial arteries 176

Calcium channel blocker 307 c-AMP 93 Cardiac output 323

- preservation 406

Carolina rinse solution 49

Catalase 117

Cathepsin 227

Cholesterol $7 \alpha$-hydroxylase 389 Cirrhotic liver 313 CK-11B 269 Closed fracture 277 Cold ischemia 285, 307 Colonic anastomosis 250 Colorectal cancer 258 Constipation 63 Coronary flow 269 Cross-clamping 323 Cyclosporine 69 L-Cysteine 363

D-dimer 57 Defecation 63 Dextrans 406

Disseminated intravascular coagulation 227

Electron microscopy 205 Encephalopathy 134 Endotoxemia 11 Endotoxin localization 216 Energy charge 77,93,313 Energy metabolism 285 Epiphysis 197 Esophageal disease 27 Experimental surgery 151 Extracorporeal rat heart model 406 Extrahepatic cholestasis 332

Fibrin glue 346 Folinicacid 69 Fracture healing 277 Free radicals 285

Gabexate mesilate 57 Gastric mucosa 27, 39

- $\quad$ substitution 27

Glutathione 332

Graft-versus-host disease 19 Growth hormone 93

Haemoglobin saturation 82 Haemorrhage 379 Heart rate 323

- transplantation 406

Heparin 184

Hepatectomy 77, 127, 241, 389 Hepatic anatomy 151

- energy metabolism 93

- protein synthesis 396

- regeneration 396

- vascular occlusion 313 Hepatobiliary system 340 Histology 205 Histopathology 27 Hormones, gastrointestinal 31

Hyaluronic acid 1 Hyperammonemia 134 Hypertonic-hyperosmotic solution 269

Hypovolaemia 379 Hypoxanthine 117

Immunity, tumor 258 Immunohistochemical staining

216 Incontinence 63 Indomethacin 117 Insulin secretion 167 Intestinal impairment 11 Intra-abdominal abscess 222

Intraosseous pressure 197 Isch(a)emia reperfusion 100,269,

379

- $\quad$ - injury 285,363

Ischemic injury, liver 313

Islets of Langerhans 167

Isolation 167

Ketamine 323 Ketone body ratio 241 Kidney functions 307 
Laser Doppler flowmetry 82

- photometry 82

Leukotrienes 117

Limulus clotting system 216

Lipid peroxidation 332

Lipids 222

Liposome 301 Liver 145

- blood flow 1

- function 205

- graft 353

- $\quad$ isch(a)emia 69

- $\quad$-, reperfusion 82

- metastases 301

- mitochondria 313

- regeneration 69,127

- $\quad$ surgery 57

- tissue adenosine-5' -triphos-phate 313

- weight 389

Local passive transfer 189 Low molecular weight heparin

184 Lung 363

- transplantation 176

Lymph node dissection 346

427

Lymphocyte 241 Lymphorrhea 346

Macrophages procoagulant

activity 222 Mebendazole 340 Membrane potential 145 Metabolism 39 Microcirculation 39 Mitochondria 77, 145 Molecular weight 406 Monoclonal antibody against

factor C, murine 216 MTT assay 241 Myocardial reperfusion injury

269

Nanocoll ${ }^{\mathrm{TM}} 137$ Nicardipine 307

Octreotide 371 ONO3708 100

Orthotopic rat liver transplantation 285 Oxygen consumption 82

- delivery 292

- extraction 292

- free radicals 292

- radicals 117

- $\quad$ supply 82

Pancreatic juice 371 Pancreatitis, biliary 137 Pancreatoduodenectomy 371 Pantothenic acid 158 Partial hepatectomy 396 - orthotopic liver transplanta

tion 205

Perifusion 167 Peritonitis 1

Permeability 406

Phagocytosis 137

Phospholipase $\mathrm{A}_{2} 332$

Plasmin 57

Platelet 77

Platelet-activating factor antagonist 379

Polymorphonuclear neutrophils 184,189

Portacaval shunt 134

Portal venous stasis 134

Postoperative management 57

Prostaglandin L 100

Proteasome 227

Protein metabolism 227 
Proton magnetic resonance imaging 353

Pulmonary circulation 117

Purification 167

Reactive oxygen radicals 189 REC4-1 49 REC16-11 49 Reduced-size graft 151 Regional blood flow 292 Reperfusion injury 49 Resection, intestinal 31 Reticuloendothelial system 137 -- function 127 Rhodamine123 145

Sacral nerves 63

Scintigraphy 137

Secretion, gastric 31

Sepsis 1,241

Septic shock 1

Serum $7 \alpha$-hydroxycholesterol 389

Shock 379

Skeletal muscle 227

Skin 158

Small bowel transplantation 19 Sodium nitroprusside 323 Somatostatin 371 Spin-lattice relaxation time 353 Spin-spin relaxation time 353 Staphylococcal sepsis 189 Stroke volume 323 Superoxide 184

- dismutase 69 Surgical anatomy 151

- damage 19

T-cell subsets 258 Thermodilution 323 Thiobarbituric-acid-reactive

species 332 Thioredoxin, human 363 Thoracic aorta 323 Thrombin 57 Thromboxane A2 100

- - receptor antagonist 100

Trace element 158

Transplantation 151,167,363

Tumor necrosis factor 189

Tumor-infiltrating lymphocytes

258

Viability 353 Vitamin 158

Warm ischemia 285 Water molecules 353 Wound healing 250

- - process 158,346

Xanthine oxidase 117

428

Subject Index 